\title{
Characterization of IK cytokine expression in mouse endometrium during early pregnancy and its significance on implantation
}

\author{
RUYUE SHAO, XUEQING LIU, YUBIN DING, XUEMEI CHEN, RUFEI GAO, JUNLIN HE and YINGXIONG WANG
}

Laboratory of Reproductive Biology, Chongqing Medical University, Yuzhong, Chongqing 400016, P.R. China

Received March 5, 2012; Accepted April 17, 2012

DOI: $10.3892 /$ ijmm.2012.1019

\begin{abstract}
The expression of IK cytokine was investigated in the mouse endometrium during early pregnancy (D1-D7 of pregnancy) and pseudopregnancy using real-time PCR, western blotting and immunohistochemical analysis, and the effects of IK cytokine on embryo implantation were observed by injection with antisense IK cytokine oligodeoxynucleotides in the uterine horn. Our data showed that the expression of IK cytokine mRNA increased gradually from D1 to D4 of pregnancy and reached a peak level at $\mathrm{D} 4$ of pregnancy $(\mathrm{P}<0.05)$. Western blotting and immunohistochemical analysis revealed that the expression of IK cytokine protein increased gradually from D1 to D5 of pregnancy and reached a peak level at D5 of pregnancy $(\mathrm{P}<0.05)$. The expression of IK cytokine in the pseudopregnant uterus was significantly lower compared to that in the normal pregnant uterus and the level of the protein never showed a high peak during the whole pseudopregnancy. The expression of IK cytokine at the implantation site was much stronger than that in the peri-implantation site on Day 5 of pregnancy. After 24 and $48 \mathrm{~h}$ of injection with antisense IK cytokine oligodexynucleotides in the uterine horn on D3 of pregnancy (i.e. implantation window), the expression of IK cytokine in the uterus was remarkably inhibited, while the expression of major histocompatibility complex II (MHCII) increased and the number of implanted embryos significantly decreased in the site of uterine horns receiving antisense IK cytokine $(\mathrm{P}<0.05)$. These results suggested that IK cytokine may play a crucial role in implantation.
\end{abstract}

\section{Introduction}

Successful implantation is very important for the pregnancy of species. It is not only a complex and intricate cross-talk between the embryonic and maternal tissues, but also an abso-

Correspondence to: Dr Yingxiong Wang or Dr Junlin He, Laboratory of Reproductive Biology, Chongqing Medical University, 1 Yixueyuan Road, Yuzhong, Chongqing 400016, P.R. China

E-mail:wyx61221@yahoo.com.cn

E-mail: hejunlin_11@yahoo.com.cn

Key words: embryo implantation, IK cytokine, immune tolerance, major histocompatibility complex II lute requirement for the development of embryos (1,2). A series of stages are included in the process of implantation: a normal and functional embryo which develops to the blastocyst stage, maternal endometrium during the implantation window (ready to accept the blastocyst), trophoblast adhesion, penetration and invasion. The coordination of these processes lie with the regulation and interplay of several factors including prostaglandins, leukotrienes, cytokines and growth factors, in which cytokines play a critical role (3).

Cytokines are small multifunctional cell-signaling glycoproteins that are secreted by numerous cells of the immune system and other systems of the body and have pleiotropic regulatory effects on endocrine, hematopoietic, nervous and immune systems. Cytokines can be classified as proteins, peptides, or glycoproteins, such as interleukins, interferons and tumor necrosis factors, which play a crucial role in the initiation, development and regulation of the immune response (4). However, as molecules, cytokines are not only limited to their immunomodulatory role, but also involved in several developmental processes during embryogenesis and embryo-maternal interactions $(5,6)$. Entry of the blastocyst into the receptive uterine is able to encourage trophoblastic cells and uterine epithelium to produce cytokines that can regulate the endometrial receptivity by modulating the expression of various adhesion molecules $(6,7)$. Failure of mammalian implantation and defective placental formation sometimes result from decreased expression of cytokines and their signaling (8).

IK cytokine, a $19 \mathrm{kDa}$ factor coded by the IK gene localized on chromosome 2p15-p14 (9), was first isolated and purified from the conditioned culture medium of the leukemic cell line K562. It was reported that IK cytokine inhibited class II major histocompatibility complex (MHCII) constitutive expression $(10,11)$. One study demonstrated that IK cytokine modulated HLA-DR expression on hematopoietic cells and has an effect on growth factor-dependent $\mathrm{CD} 34^{+}$cell proliferation and differentiation by regulating HLA-DR expression (12). It is also accepted that the 'semi-foreign' or semiallogeneic fetus can avoid maternal immune attack during normal pregnancy. Although the mechanism underlying the 'tolerant' state of the mother throughout pregnancy has not been clearly explained, the absence of trophoblast MHCII antigen expression is thought to be an important strategy for ensuring the maintenance of normal pregnancy (13). IK cytokine has close relationship with the expression of MHCII. Up to now, there are no relevant articles addressing whether IK cytokine plays 
a role in the fetomaternal immune tolerance during pregnancy. Thus, this study examined IK cytokine expression in mouse uterus of early pregnancy confirmed its role in implantation, and provided theoretical evidence for the mechanism of implantation and preventive and therapeutic methods of pregnancy failure.

\section{Materials and methods}

Materials. Ethics approval for this study was granted from our local Ethics Committee. Adult mice of the Kunming white strain (8-10 weeks of age and weighing 25-30 g) were obtained from the Experimental Animal Center of Chongqing Medical University [certificate no. SCXK(YU)2007-0001]. The mice were maintained in specific pathogen-free (SPF) room with a controlled light schedule (14L:10D) and a controlled temperature range $\left(22-25^{\circ} \mathrm{C}\right)$ with free access to food and water. All animal procedures were approved by the Ethics Committee, Chongqing Medical University, China. The adult female mice were mated with fertile males (female:male, 2:1) overnight $(12 \mathrm{~h})$ and vaginal plugs were checked the next morning. The day of positive vaginal plus was considered as Day 1 of pregnancy (D1). In addition, some male mice were subjected to vasoligation. Two weeks later, they were caged with females (female:male, 2:1) overnight to induce preudo-pregnancy (PD1, PD1 = the day of vaginal plug positive).

Analysis of IK cytokine expression in mouse endometrium during early pregnancy. Total-RNA from approximately 50-100 mg mouse endometrial tissues of D1 to D7 and PD1 to PD7 of pregnancy were extracted using TRIzol reagent (Invitrogen) following manufacturer's instructions. RNA concentration was determined by spectrophotometric measurement the ratio of $A_{260} / A_{280}$. The expression of IK cytokine in the mouse endometrium of D1 to D7 were detected by real-time PCR, western blotting and immunohistochemical analysis. The expression of this protein in mouse endometrium of PD1 to PD7 were examined using immunohistochemical analysis. The expression of IK cytokine at implantation site and inter-implantation site of D5 were detected with western blotting.

Real-time PCR analysis. Primer sequences of IK cytokine (NCBI no. NM_011879) and $\beta$-actin (synthesized by Sangon Biotech Co., Ltd., Shanghai, China) are as follows: IK cytokine, sense, ACGCAGAATGCTATCC and antisense, CAGA GCCTCCTTGTTGT; $\beta$-actin, sense, CCTGAGGCTCTTTT CCAGCC and antisense, TAGAGGTCTTTACGGATGTC AACGT. Real-time PCR was carried out by Bio-Rad CFX 96 ${ }^{\mathrm{TM}}$ using SYBR-Green (Takara code: DRR081A). cDNA of $100 \mathrm{ng}$ template was added to $12.5 \mu 1$ of $2 \mathrm{X}$ SYBR Premix Ex Taq and $0.5 \mu 1$ of each specific primer, and water to a final volume of $25 \mu \mathrm{l}$. The reactions were performed for 40 cycles according to the following parameters: denaturation at $95^{\circ} \mathrm{C}$ for $30 \mathrm{sec}$; annealing at $95^{\circ} \mathrm{C}$ for $10 \mathrm{sec}$; and extension at $56^{\circ} \mathrm{C}$ for $30 \mathrm{sec}$. The fluorescence signal was plotted at the end of the extension phase of each cycle. Melting curve analysis was performed by the instrument automatically. This experiment was repeated 3 times. To determine a normalized arbitrary value for each gene, $\beta$-actin was used as the reference gene.

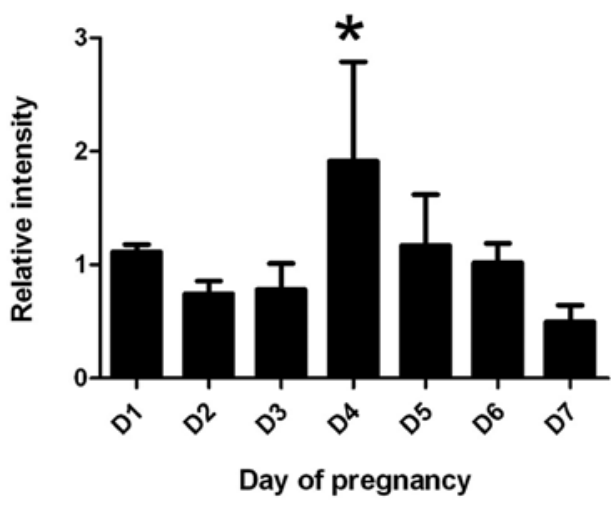

Figure 1. Real-time PCR analysis of IK cytokine mRNA levels in mouse endometrium during early pregnancy. The experiment was performed three times. Three mice of each day of pregnancy were used in each experiment. Statistical significance $(* \mathrm{P}<0.05)$ between D4 and all the other days of pregnancy.

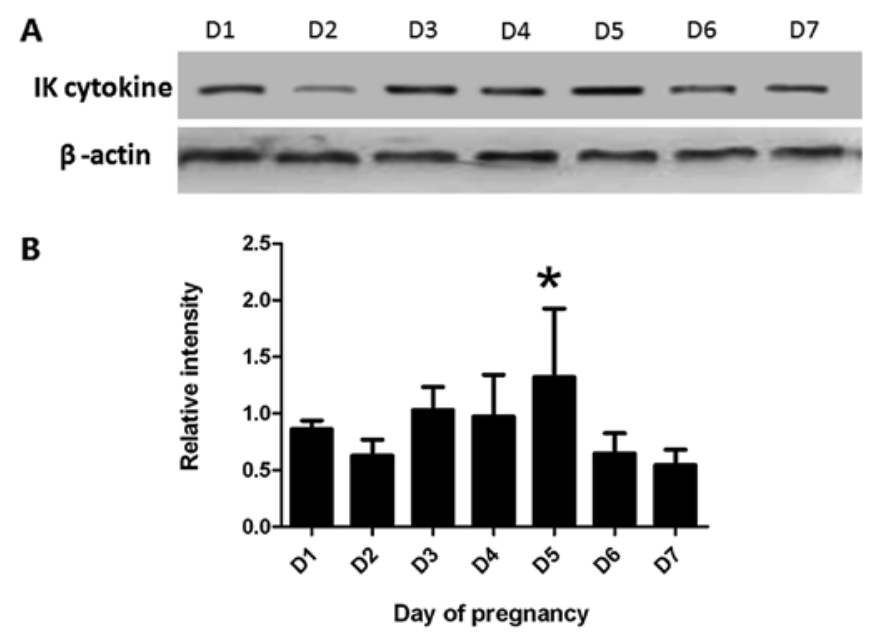

Figure 2. Western blot analysis of IK cytokine protein in mouse endometrium during early pregnancy. (A) Western blot analysis electrophoresis graph. (B) Relative densitometric analysis of IK cytokine protein during pregnancy D1-D7. A single representative experiment is shown. The experiment was performed in triplicate. Three mice of each day of pregnancy were used for the experiment. Statistical significance $\left({ }^{*} \mathrm{P}<0.05\right)$ between $\mathrm{D} 5$ and all the other days of pregnancy.

Relative expression was calculated according to the equation $2^{-\Delta \Delta C t}$ and statistically analyzed by t-test.

Immunohistochemical analysis. Performed according to the SP-9001 Reagent kit (Zhongshan Goldenbridge Biotechnology Co., Ltd.). The sections were observed and phototgraphed under a microscope (Olympus BX51). The intensity of positive expressions were determined by Image Pro $^{\circledR}$ Plus v.6.0 software. Non-immune serum was used instead of the primary antibody for negative control.

Western blot analysis. Approximate $100 \mathrm{mg}$ mouse endometrial tissues were homogenized in $200 \mu 1$ lysis buffer (Beyotime, China) and $2 \mu 1$ chymostatin PMSF (100 mmol/l). Total protein was extracted from the mouse endometrium of each group using the total protein TriPure reagent kit (Takara Bio, Inc., China) according to the manufacturer's instructions. The protein concen- 

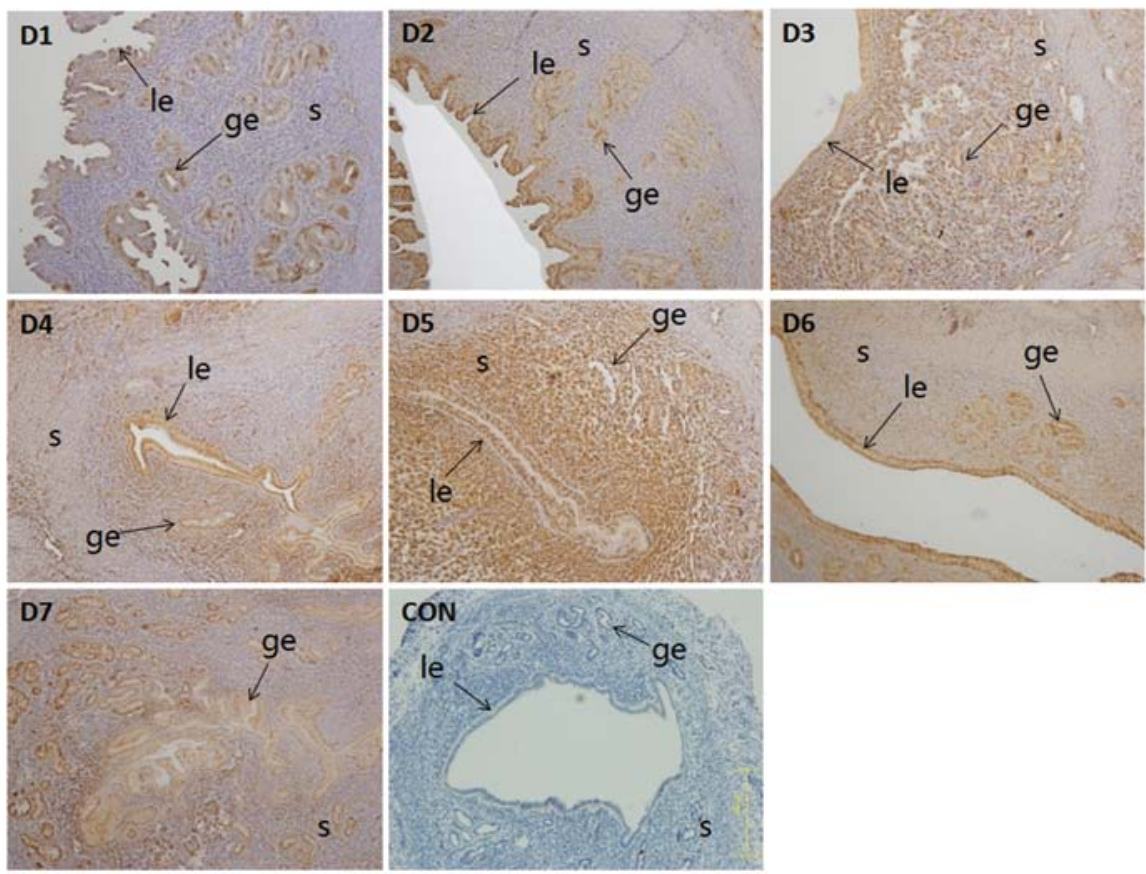

Figure 3. Immunohistochemistry of IK cytokine in mouse endometrium during early pregnancy. A single representative experiment is shown; the experiment was performed three times. Three mice of each day of pregnancy were used in each experiment. le, luminal epithelium; ge, glandular epithelium; s, stroma; CON, negative control; bar, $200 \mu \mathrm{m}$.

tration was examined by the Bradford assay. Proteins samples (about $50 \mu \mathrm{l}$ each sample) were subjected to $8 \%$ SDS-PAGE gels and then transferred onto PVDF membranes by a Bio-Rad electroblot apparatus (Bio-Rad, Beijing, China). The membrane was incubated in blocking PBS containing $0.05 \%$ Tween-20 (TBST) and 5\% nonfat milk for $1.5 \mathrm{~h}$ at room temperature and then was subsequently incubated with monoclonal rabbit anti-IK cytokine antibody (1:500, Santa Cruz Biotechnology, Inc., IK (G-13): sc-135485) and $\beta$-actin (1:500, Zhongshan Goldenbridge Biotechnology Co., Ltd., TA-09) at $4^{\circ} \mathrm{C}$ for $12 \mathrm{~h}$, washed for three times with PBST (5 min each time), following by incubation with secondary antibody (goat anti-rabbit IgG) conjugated with horseradish peroxidase (Zhongsan Biosciences, Inc., Beijing, China) for $2 \mathrm{~h}$ at room temperature, and then washed three times with PBST (5 min each time). Protein bands were visualized using diaminobenzidine tetrahydrochloride (DAB). Densitometry analysis of IK cytokine expression was performed by Quantity One version 4.4.0 software. The $\beta$-actin protein was used as the internal control.

Uterine horns injection of IK cytokine oligodeoxynucleotides. Ninety female mice were given a uterine horn injection under $3 \%$ pentobarbital sodium anesthetic on D3 according to the procedures described by Zhu et al (14). Among the 90 mice, 60 were injected with $10 \mu \mathrm{g}$ antisense IK cytokine oligodeoxynucleotides (IK cytokine A-ODNs, diluted in $100 \mu \mathrm{l}$ double-distilled water) in the left of uterine horns and equal volum of sense IK cytokine oligodeoxynucleotides (IK cytokine S-ODNs) in the right of the uterine horns. The remaining 30 mice were injected with equal volume of distilled water in both uterine horns as control. Fifteen mice receiving respectively injection of IK cytokine A-ODNs and IK cytokine S-ODNs in the both horns were killed after $24 \mathrm{~h}$ of injection (D4) and another
15 mice with the same treatment were sacrificed after $48 \mathrm{~h}$ of injection (D5), the tissues of endometrium were collected for the detection of expression of IK cytokine and MHCII antigen by immunohistochemical and western blot analysis. Another 30 receiving respectively injection of IK cytokine A-ODNs and IK cytokine S-ODNs and 30 mice which were injected in both horns with distilled water were sacrificed on D7 and the number of implanted embryos was recorded in each uterine horn. The ODNs were designed and synthesized by Biosune Co., Ltd. (Shanghai, China). The sequence of the IK cytokine A-ODNs was, 5'-GTGAGCCCTTCTCTAACCCT-3'-FITC; and the sequence of IK S-ODNs was 5'-AGGGTTAGAGAAG GGCTCAC-3'-FITC. To ensure their long half-lives in cells, the sequences were thiophosphate-modified. MHCII antigens (NCBI no. XM_003457013) (synthesized by Sangon Biotech Co., Ltd.) were as follows: MHCII sense: AAGAA GGAGACTGTCTGGATGC and antisense: TGAATGATGAA GATGGTGCCC.

Statistical analysis. All statistical data obtained from the experiment were analyzed using SPSS software (version 11.5), and t-test was used to analyze the data. Differences were considered significant at $\mathrm{P}<0.05$.

\section{Results}

Expression of IK cytokine $m R N A$. By real-time PCR, the expression of IK cytokine mRNA increased gradually from D1 to D4 in mouse endometrium and reached a peak level at D4 $(\mathrm{P}<0.05)$, and then decreased gradually from D5 to D7 (Fig. 1).

Expression of IK cytokine protein. By western blotting, the expression of IK cytokine protein increased gradually from D1 
A

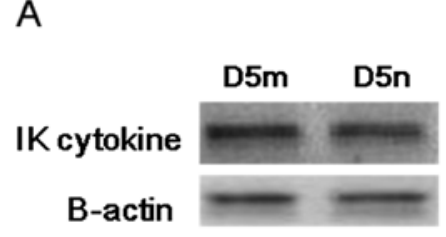

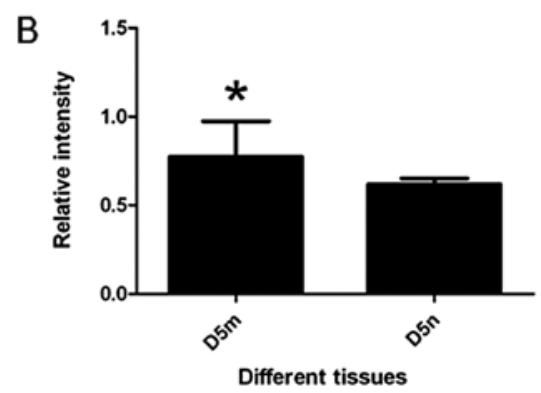

Figure 4. Comparison of IK cytokine protein expression in mouse endometrium between the implantation site and the inter-implantation segment. A single representative experiment is shown; the experiment was repeated three times. (A) Western blotting electrophoresis graph. (B) Relative densitometric analysis of IK cytokine protein between the implantation site (D5m) and the inter-implantation segment (D5n).
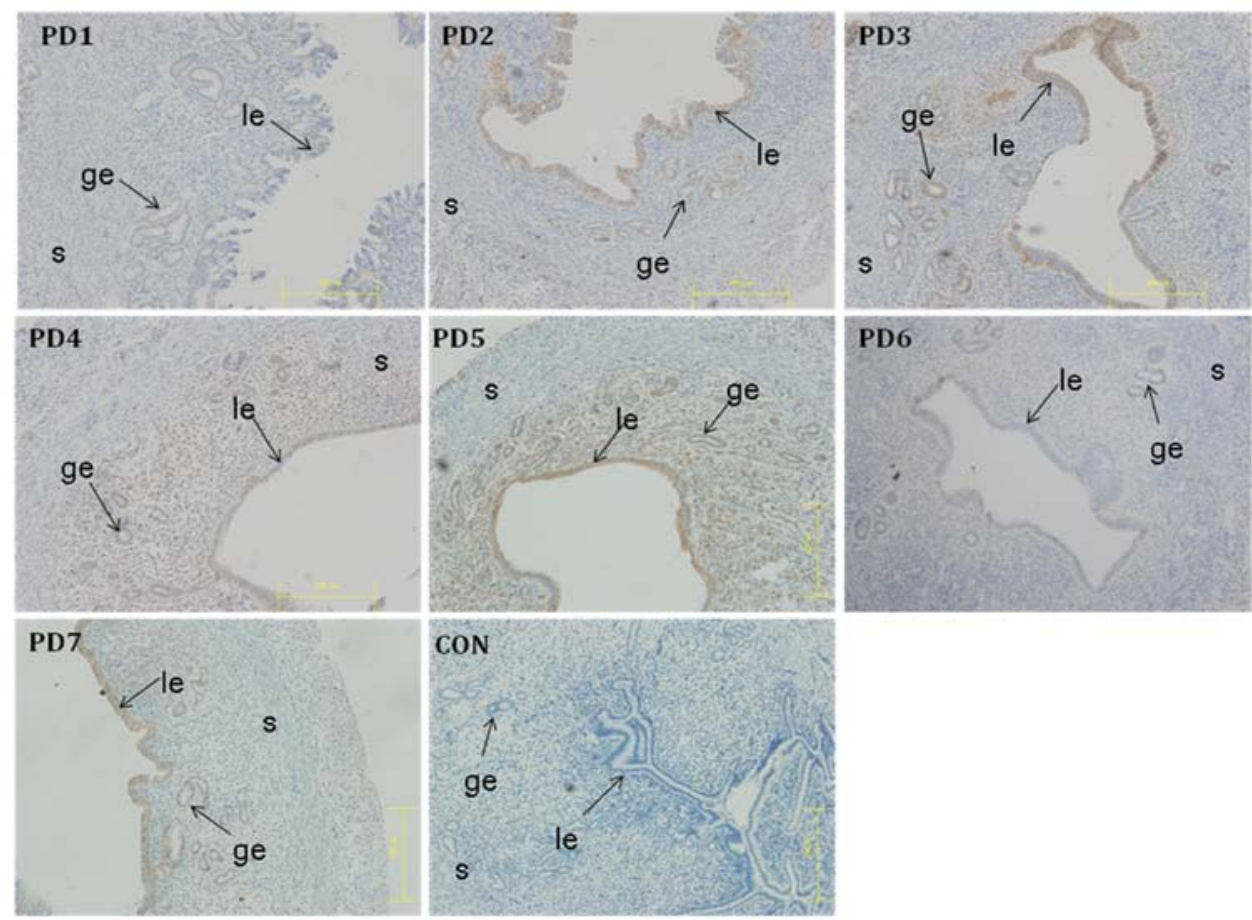

Figure 5. Immunohistochemistry of IK cytokine in mouse endometrium during pseudopregnancy. A single representative experiment is shown; the experiment was repeated three times. Three mice of each day of pregnancy were used in each experiment. le, luminal epithelium; ge, glandular epithelium; s, stroma; CON, negative control; bar, $200 \mu \mathrm{m}$.

to D5 in mouse endometrium and reached a peak level at D5 $(\mathrm{P}<0.05)$, and then decreased gradually from D6 to D7 (Fig. 2). The change of IK cytokine protein expression coincided with the mRNA levels detected by real-time PCR.

Location of IK cytokine expression. By immunohistochemical analysis, the weak expression of IK cytokine was observed and mainly localized in the luminal and the glandular epithelium on D1 and D2. The highest expression occured on D3 to D5 in the luminal epithelium, glandular epithelium and stromal cells, following by decreasing gradually from D6 to D7 (Fig. 3). The change of IK cytokine expression coincided with the mRNA levels detected by real-time PCR.

Expression of IK cytokine protein in endometrium at the implantation site and the inter-implantation site. By western blotting, IK cytokine expression at the implantation site was significantly stronger than that at the inter-implantation site on D5 $(\mathrm{P}<0.05)$ (Fig. 4).

Expression of IK cytokine in mouse endometrium during pseudopregnancy. The expression of IK cytokine in mouse endometrium on PD1 to PD7 were analyzed by immunohistochemical analysis. The protein was weakly expressed on PD1, increased slightly on PD2 to PD3, sharply decreased on PD4 in the luminal and the glandular epithelium, with a remaining low level on PD5 to PD7. The expression of the protein never reached a peak in the mouse endomentrium during pseudopregnancy (Fig. 5).

Inhibition of IK cytokine expression in the endometrium of pregnant mice receiving IK cytokine A-ODNs and effect on embryo implantation. Mice uterine horns received injection of IK cytokine A-ODNs, IK cytokine S-ODNs and distilled 

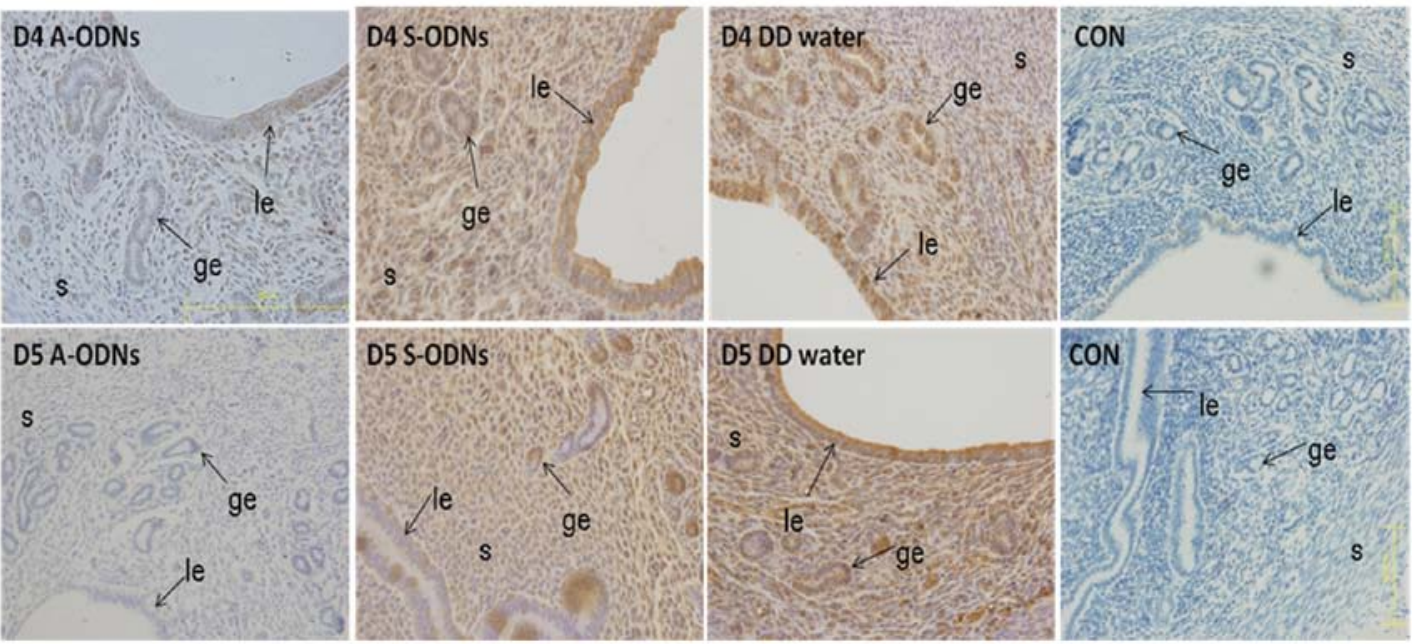

Figure 6. Immunohistochemistry of IK cytokine protein expression suppressed by antisense ODNs. A single representative experiment is shown; the experiment was repeated three times. Three mice of each day of pregnancy were used in each experiment. S-ODNs, sense oligodeoxynucleotides; A-ODNs, antisense oligodeoxynucleotides; DD water, double distilled water; le, luminal epithelium; ge, glandular epithelium; s, stroma; CON, negative control; bar, $200 \mu \mathrm{m}$.

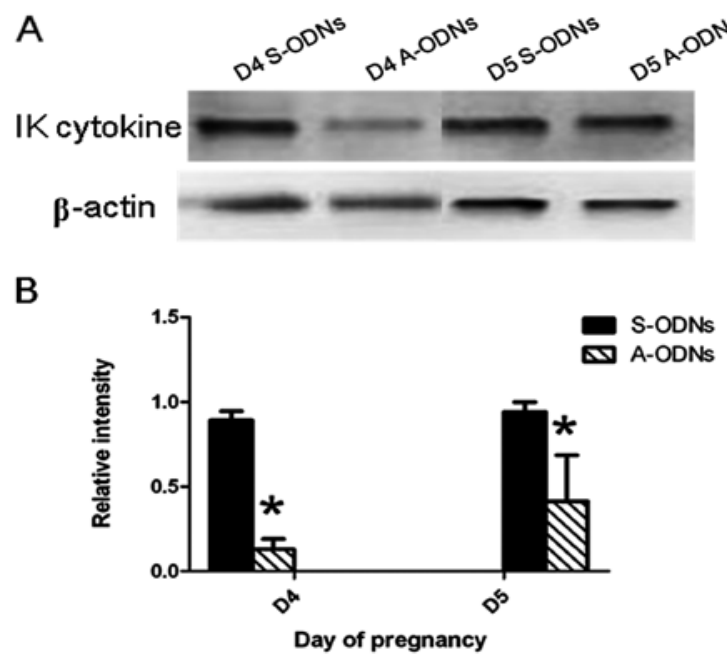

Figure 7. Suppression of IK cytokine protein expression by antisense ODNs. (A) Western blot analysis electrophoresis graph. (B) Relative densitometric analysis of IK cytokine protein before and after intrauterine injection of ODNs.

water on D3. The expression of IK cytokine assessed by immunohistochemistry was sharply inhibited on the side of the uterine horns receiving IK cytokine A-ODNs after 24 and $48 \mathrm{~h}$ (i.e. D4 and D5) $(\mathrm{P}<0.05)$. In contrast, the expression of this protein was not changed in the control groups which were injected with IK cytokine S-ODNs or distilled water (Fig. 6). The results from western blot analysis were similar to those of the immunohistochemical analysis (Fig. 7). This indicated that the expression of IK cytokine was indeed inhibited by IK cytokine A-ODNs.

The mice, which received either injection of IK cytokine A-ODNs or IK cytokine S-ODNs or distilled water in the uterine horns on D3, were sacrificed on D7 under anesthesia. The number of implanted embryos in each uterine horn was recorded by dissection. The number of implanted embryos was sharply reduced in the A-ODNs-treated group $(\mathrm{P}<0.05)$, but no embryo abnormality was observed. In contrast, no significant

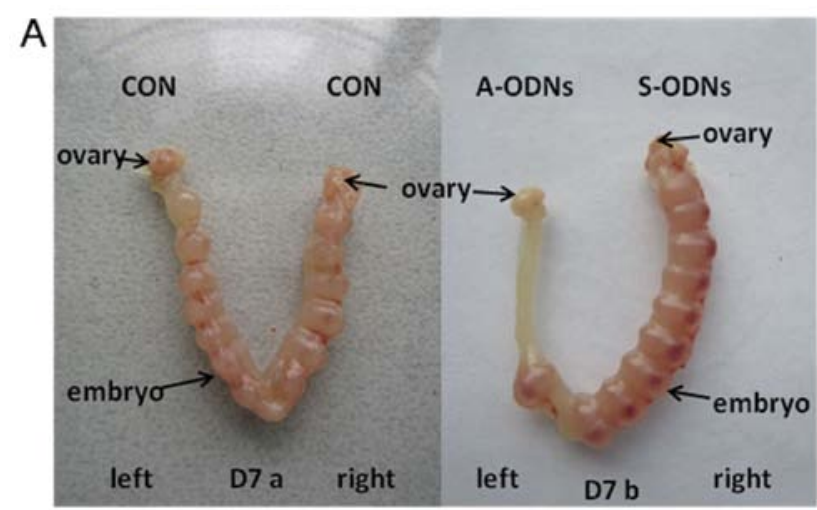

B

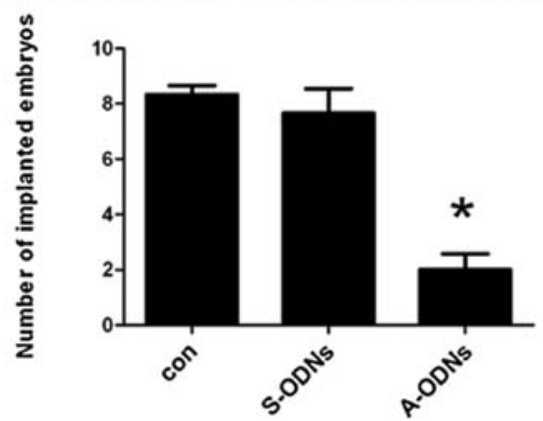

Figure 8. Effect of antisense oligodeoxynucleotide on embryo implantation. (A) Two representative uteri of D7 (n=60). (D7a) Control group (CON) $(n=30)$, pregnant mouse was injected in both horns with DD water. (D7b) Treatment group $(n=30)$, pregnant mouse was injected with IK cytokine A-ODNs in the left horn, with IK cytokine S-ODNs in the right horn on Day 3 of pregnancy. (B) Statistical analysis of implanted embryo numbers in the uteri with different treatments $\left({ }^{*} \mathrm{P}<0.05\right)$.

change in the number of implanted embryos was observed on the side of the uterine horns of mice receiving IK cytokine S-ODNs and those receiving the distilled water which were used as the controls (Fig. 8A). At the same time, all the embryos in the S-ODNs-treated horns had a normal appearance and size as compared with those in the water-treated control group, implying that both the ODNs itself and the dose used in this study was non-toxic to embryo implantation. 

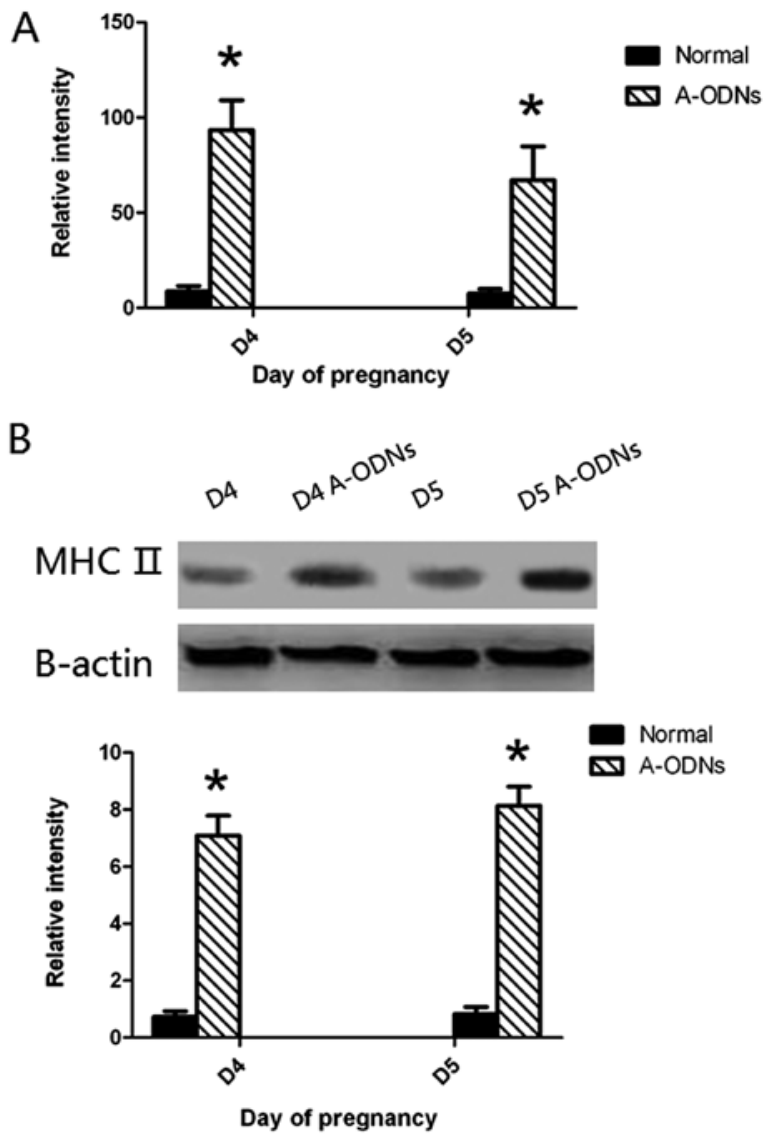

Figure 9. Effect of antisense oligodeoxynucleotide on MHCII expression. (A) Real-time PCR analysis of MHCII expression in mouse uterus during early pregnancy. (B) Western blotting detection of MHCII expression in the mouse uterus during early pregnancy.

Effect of IK cytokine A-ODNs on the expression of MHCII in mouse endometrium during early pregnancy. To understand whether MHCII expression in mouse endometrium of the implantation window would be influenced after IK cytokine expression was suppressed by IK cytokine A-ODNs, MHCII expression were detected by real-time PCR, western blotting before and after injection of IK cytokine A-ODNs. This result revealed that MHCII expression in the mouse endometrium was much stronger 24 and $48 \mathrm{~h}$ after receiving injection of IK cytokine A-ODNs $(\mathrm{P}<0.05)$ (Fig. 9).

\section{Discussion}

Embryonic implantation is a complicated process which needs the coordination between a blastocyst capable of implanting and an endometrium receptive to the embryo. A variety of molecules produced by embryonic and maternal tissues play a critical role in the regulation of the process (15-17). The endometrium is receptive to the embryo during only a limited and restricted phase (D4-D5 of pregnancy) referred to as the window of implantation $(18,19)$. Certain molecules are expressed during the window of implantation and a number of them were recognized as biochemical markers of uterine receptivity (20-22). This study indicates that the expression of IK cytokine in the mouse endometrium during early pregnancy is time-dependent. The expression of IK cytokine
mRNA increased gradually from D1 to D4 and reached a peak level on D4. Western blot analysis and immunohistochemistry also showed that its protein expression increased from D1 to D5 and reached a peak level on D5, coinciding with the expression of IK cytokine mRNA on space-time. Additionally, IK cytokine expression at the implantation sites was much higher than that at the inter-implantation site. However, the expression IK cytokine protein in pseudopregnant mouse endometrium was markedly lower than that in normal pregnant status and no peak level was observed during the whole pseudopregnant period. It was known that oligodeoxynucleotide can be effectively absorbed by the uterine luminal and glandular epithelium and stroma cells (23). Antisense oligodeoxynucleotide is known to have a half life of $24-48 \mathrm{~h}$ in certain tissues (24). Therefore, one may anticipate that the IK A-ODNs which were injected into the uterine horns on D3 can survive for the subsequent D4 and D5 and remain effective for suppression of IK expression. In this study, the performance of injecting IK cytokine A-ODNs into the uterine horns on D3 led to suppressed expression of IK cytokine in the endometrium after 24 and $48 \mathrm{~h}$, and in a reduced number of implanted embryos. The characteristic of IK cytokine expression at the implantation window and implantation sites as well as the reduction of implanted embryos due to the inhibition of IK cytokine expression strongly imply that IK cytokine may be essential for embryo implantation.

The conception of 'the fetus as a semiallogeneic graft' was proposed by Medawar in the early 1950s because the mammalian fetus can express paternally derived polymorphic antigens. As a result, the mammalian fetus is potentially at risk of attack by the maternal immune system during pregnancy. This kind of immunological situation also exist in transplantation rejection reactions (25). However, the fetus is tolerant of the maternal tissues during the whole period of gestation. The immunological interplay between the embryonic and maternal tissues depend on the antigens which are expressed on the surface of the fetus and the maternal recognition and reaction to them. A series of mechanisms may participate in modulating maternal immune tolerance throughout gestation, among which the expression of the major histocompatibility complex (MHC) antigen was considered to play a critical role (13). Class II MHC antigens are expressed constitutively on the surface of antigen-presenting cells (APCs) such as dendritic cells, macrophages and B cells. It has been proposed to be critically important for the initiation, development and modulation of the immune response (11). In addition, MHCII expression can be induced in the majority of cells by numerous cytokines, especially by interferon- $\gamma($ IFN- $\gamma)(26)$. It has been reported that aberrant expression of MHCII antigens was observed in the target organs of autoimmune diseases and its expression level correlated closely with the intensity of the immune responses $(27,28)$. The inhibition of MHC antigen expression in trophoblasts is crucial for the evasion of rejection by the maternal immune system to the fetus with paternal antigens and for the maintenance of successful pregnancy (29). Trophoblast cells lack constitutive expression of MHCII antigens and cannot be induced to express MHCII molecules by IFN- $\gamma(13,29)$. Inappropriate MHCII expression has been reported to be recognized in trophoblasts from the placentas of women suffering abortion (30-32). These facts demonstrated 
that the suppression of MHCII antigens was crucial for the maintenance of successful pregnancy. It has been confirmed that IK cytokine can inhibit expression of MHCII antigen $(10,11)$. In the present study, we found that the expression of IK cytokine in the mouse endometrium reached a peak level at 'implantation window', but a significant reduction of its protein expression and a remarkable increase of MHCII expression were observed on D4 and D5 (implantation window) after treatment with IK A-ODNs on D3. A significant decrease of the number of implanted embryos was also found. These findings suggest that the suppression of MHCII antigens by IK cytokine was likely to inhibit the fetal-maternal immune response, contributing to maternal immune tolerance of conceptus and the maintenance of successful pregnancy. When the expression of MHCII is no longer suppressed because of the inhibition of IK cytokine expression, it will result in attacking maternal immune system will elicit a response against the fetus which will influence embryo implantation. However, embryo implantation has not been completely inhibited by suppressing the expression of IK cytokine, implying involvement of certain other mechanisms participating in the regulation of fetal-maternal immune tolerance, which remains to be further investigated.

Our data have revealed characterization of IK cytokine expression in the mouse endometrium during early pregnancy and its significance an embryo implantation. However, the molecular mechanism of IK cytokine in modulating fetalmaternal immune tolerance and embryo implantation remains to be further examined.

\section{Acknowledgements}

This study was supported by grants from the National Natural Science Foundation of China (nos. 31071278 and 30973195) and the Scientific Research Foundation of Chongqing Science and Technology Commission (CSTC 2009BA5082).

\section{References}

1. Garrido-Gómez T, Dominguez F and Simón C: Dioxins: a novel regulator in embryo implantation. Sci World J 11: 235-241, 2011.

2. Guo B, Tian Z, Han BC, Zhang XM, Yang ZM and Yue ZP: Expression and hormonal regulation of Hoxa10 in canine uterus during the peri-implantation period. Reprod Domest Anim 44: 638-642, 2009

3. Madeja Z, Yadi H, Apps R, Boulenouar S, Roper SJ, Gardner L, Moffett A, Colucci F and Hemberger M: Paternal MHC expression on mouse trophoblast affects uterine vascularization and fetal growth. Proc Natl Acad Sci USA 108: 4012-4017, 2011.

4. Cannon JG: Inflammatory cytokines in nonpathological states. News Physiol Sci 15: 298-303, 2000.

5. Saito S: Cytokine cross-talk between mother and the embryo/ placenta. J Reprod Immunol 52: 15-33, 2001.

6. Singh M, Chaudhry P and Asselin E: Bridging endometrial receptivity and implantation: network of hormones, cytokines, and growth factors. J Endocrinol 210: 5-14, 2011.

7. Simón C, Martín JC and Pellicer A: Paracrine regulators of implantation. Baillieres Best Pract Res Clin Obstet Gynaecol 14: $815-826,2000$.

8. Guzeloglu-Kayisli O, Kayisli UA and Taylor HS: The role of growth factors and cytokines during implantation: endocrine and paracrine interactions. Semin Reprod Med 27: 62-79, 2009.

9. Krief P, Augery-Bourget Y, Plaisance S, Merck MF, Assier E, Tanchou V, Billard M, Boucheix C, Jasmin C and Azzarone B: A new cytokine (IK) down-regulating HLA class II: monoclonal antibodies, cloning and chromosome localization. Oncogene 9: 3449-3456, 1994.
10. Vedrenne J, Assier E, Pereno R, Bouzinba-Segard H, Azzarone B, Jasmin C, Charron D and Krief P: Inhibitor (IK) of IFN-gamma induced HLA class II antigens expression also inhibits HLA class II constitutive expression in the human Raji B cell line. Oncogene 14: 1453-1461, 1997.

11. Muraoka M, Hasegawa $H$, Kohno M, Inoue A, Miyazaki $T$, Terada M, Nose M and Yasukawa M: IK cytokine ameliorates the progression of lupus nephritis in MRL/lpr mice. Arthritis Rheum 54: 3591-3600, 2006

12. Cao LX, Le Bousse Kerdiles MC, Clay D, Oshevski S, Jasmin C and Krief P: Implication of a new molecule IK in CD34 ${ }^{+}$hematopoietic progenitor cell proliferation and differentiation. Blood 89: 3615-3623, 1997.

13. Murphy SP and Tomasi TB: Absence of MHC class II antigen expression in trophoblast cells results from a lack of class II transactivator (CIITA) gene expression. Mol Reprod Dev 51: 1-12, 1998.

14. Zhu LJ, Bagchi MK and Bagchi IC: Attenuation of calcitonin gene expression in pregnant rat uterus leads to a block in embryonic implantation. Endocrinology 139: 330-339, 1998.

15. Tabibzadeh $S$ and Babaknia A: The signals and molecular pathways involved in implantation, a symbiotic interaction between blastocyst and endometrium involving adhesion and tissue invasion. Hum Reprod 10: 1579-1602, 1995.

16. Makrigiannakis A and Minas V: Mechanisms of implantation. Reprod Biomed Online 1: 75-82, 2007.

17. Garrido-Gómez T, Dominguez F and Simón C: Proteomics of embryonic implantation. Handb Exp Pharmacol 198: 67-78, 2010.

18. Yoshioka S, Fujiwara H, Nakayama T, Kosaka K, Mori T and Fujii S: Intrauterine administration of autologous peripheral blood mononuclear cells promotes implantation rates in patients with repeated failure of IVF-embryo transfer. Hum Reprod 21: 3290-3294, 2006.

19. Giudice LC: Potential biochemical markers of uterine receptivity. Hum Reprod 14 (Suppl 2): S3-S16, 1999.

20. Salamonsen LA, Nie G, Dimitriadis E, Robb L and Findlay JK: Genes involved in implantation. Reprod Fertil Dev 13: 41-49, 2001.

21. Tapia A, Vilos C, Marín JC, Croxatto HB and Devoto L: Bioinformatic detection of E47, E2F1 and SREBP1 transcription factors as potential regulators of genes associated to acquisition of endometrial receptivity. Reprod Biol Endocrinol 27: 9-14, 2011.

22. Minas V, Loutradis D and Makrigiannakis A: Factors controlling blastocyst implantation. Reprod Biomed Online 10: 205-216, 2005.

23. Yuan JX, Xiao LJ, Lu CL, Zhang XS, Liu T, Chen M, Hu ZY and Gao F: Increased expression of heat shock protein 105 in rat uterus of early pregnancy and its significance in embryo implantation. Reprod Biol Endocrinol 7: 23, 2009.

24. Wagner RW: Gene inhibition using antisense oligodeoxynucleotides. Nature 372: 333-335, 1994

25. Loke YW, and King A: Immunology of human placental implantation: clinical implications of our current understanding. Mol Med Today 3: 153-159, 1997.

26. Steimle V, Siegrist CA, Mottet A, Lisowska-Grospierre B and Mach B: Regulation of MHC class II expression by interferon- $\gamma$ mediated by the transactivator gene CIITA. Science 265: 106-109, 1994.

27. Guardiola J and Maffei A: Control of MHC class II gene expression in autoimmune, infectious, and neoplastic diseases (Review). Crit Rev Immunol 13: 247-268, 1993.

28. Cheah PL, Looi LM, Chua CT, Yap SF and Fleming S: Enhanced major histocompatibility complex (MHC) class II antigen expression in lupus nephritis. Malays J Pathol 19: 115-120, 1997.

29. Peyman JA: Repression of major histocompatibility complex genes by a human trophoblast ribonucleic acid. Biol Reprod 60: 23-31, 1999.

30. Athanassakis I, Aifantis Y, Makrygiannakis A, Koumantakis E and Vassiliadis S: Placental tissue from human miscarriages expresses class II HLA-DR antigens. Amer J Reprod Immunol 34: 281-287, 1995.

31. Lata JA, Tuan RS, Shepley KJ, Mulligan MM, Jackson LG and Smith JB: Localization of major histocompatibility complex class I and II mRNA in human first-trimester chorionic villi by in situ hybridization. J Exp Med 175: 1027-1032, 1992.

32. Chatterjee-Hasrouni S and Lala PK: MHC antigens on mouse trophoblast cells: Paucity of Ia antigens despite the presence of H-2K and D. J Immunol 127: 2070-2073, 1981. 